\title{
Hedylidae (Lepidoptera: Hedyloidea) coletados à luz a 40 metros de altura no dossel da floresta da Estação Experimental de Silvicultura Tropical em Manaus, Amazonas, Brasil
}

Gilcéia Melo LOURIDO ${ }^{1 *}$, Catarina da Silva MOTTA ${ }^{1,2}$, José Albertino RAFAEL ${ }^{1,3}$, José Wellington de MORAIS ${ }^{1,4}$, Francisco Felipe XAVIER FILHO ${ }^{1,5}$

\section{RESUMO}

Os Hedylidae constituem um grupo de lepidópteros exclusivamente neotropical com 35 espécies conhecidas em um gênero, Macrosoma Hübner, 1818. Antes classificados entre as mariposas Geometridae, agora são considerados um grupo basal e noturno de borboletas. Foram realizadas coletas mensais de janeiro a dezembro de 2004 no dossel de uma floresta em área de terra-firme, utilizando um lençol branco iluminado por luz mista de mercúrio e outra luz negra UV-BLB, instalado a 40 $\mathrm{m}$ de altura em uma torre. As coletas foram realizadas das 18:00 às 6:00 h do dia seguinte. Foram coletados 55 exemplares, a maioria machos, somente dois exemplares fêmeas pertencentes a nove espécies, uma delas provavelmente nova, a saber: $M$. hedylaria Warren (1894), M. bahiata (Felder \& Rogenhofer, 1875), M. conifera (Warren, 1897), M. heliconiaria (Guenée, 1857), M. hyacinthina (Warren, 1905), M. klagesi (Prout, 1916), M. lucivittata (Walker, 1863) M. nigrimacula (Warren, 1897) e Macrosoma sp. Os Hedylidae foram mais abundantes na estação mais chuvosa, dezembro a maio, com 65,5\% dos espécimes coletados. O horário de maior atividade foi entre 19:00 e 22 h com 80\% dos espécimes coletados.

PALAVRAS-CHAVE: Amazônia Central, atividade de vôo, coleção sistemática, dossel de floresta.

\section{Hedylidae (Lepidoptera: Hedyloidea) collected with light trap at 40 meter height over an Amazon forest canopy at Experimental Station of the Tropical Silviculture in Manaus, Amazonas, Brazil}

\begin{abstract}
The Hedylidae is a lepidopteran group exclusively neotropical, with 35 known species in the genus Macrosoma Hübner, 1818 . The family was classified as Geometridae moths but presently they are considered a basal and nocturnal group of butterflies. The collections were carried out monthly from January to December of 2004, during three nights of lunar transition third quarter moon/new moon from 6 p.m. to 6 a.m. The insects were collected over a vertical white sheet illuminated by a 250 watt mixed light and a 20 watt black-light (BLB) lamp installed in a tower at a height of $40 \mathrm{~m}$. We collected 53 male specimens and two female specimens, belonging to nine species of which one was probably new to science: $M$. hedylaria Warren (1894), M. bahiata (Felder \& Rogenhofer, 1875), M. conifera (Warren, 1897), M. heliconiaria (Guenée, 1857), M. hyacinthina (Warren, 1905), M. klagesi (Prout, 1916), M. lucivittata (Walker, 1863) M. nigrimacula (Warren, 1897) e Macrosoma sp. The hedylids were more abundant in the rainy season, December to May, with $65,5 \%$ of the collected specimens. They were collected mainly from 7:00 pm to $10: 00$ pm with $80 \%$ of the collected specimens.
\end{abstract}

KEY WORDS: Central Amazonia, flight activity, systematic collection, canopy.

\footnotetext{
1 Instituto Nacional de Pesquisas da Amazônia, Coordenação de Pesquisas em Entomologia - INPA/CPEN. Av. André Araújo, 2936 Aleixo CEP $69011-970$ *autor de correspondência: lourido@inpa.gov.br

2 Pesquisador INPA/CPEN. e-mail: motta@inpa.gov.br

3 Pesquisador INPA/CPEN. e-mail: jarafael@inpa.gov.br

4 Pesquisador INPA/CPEN. e-mail: morais@onpa.gov.br

5 Técnico INPA/CPEN. e-mail: ffelipe@inpa.gov.br
} 


\section{INTRODUÇÃO}

Hedylidae é um grupo de lepidópteros restritos à região neotropical, distribuídos desde o México Central até o Sul do Brasil. Tem um gênero, Macrosoma Hübner, 1818, para o qual são reconhecidas 35 espécies. Dessas, 21 têm registros para o Brasil e 16 para a Amazônia brasileira, sendo três com localidades-tipo no estado do Amazonas, nos municípios de Tefé (antigo Ega), Fonte Boa e São Paulo de Olivença (Scoble, 1990a, b; 1998).

Hedylidae, foi tratada ora como subfamília (Druce, 1893), ora como tribo (Prout, 1916, 1932) de Geometridae. Scoble (1986) fez uma revisão do grupo e concluiu que os Hedylidae, apesar das semelhanças com as mariposas, deveriam ser consideradas borboletas noturnas, por apresentarem características únicas, dentre estas se destacam as relacionadas com o ovo, a larva e a pupa. Após a revisão, Hedylidae passou a constituir taxonomicamente uma superfamília, Hedyloidea (Scoble, 1986).

De acordo com a descrição de Scoble (1986, 1990b) e Aiello (1992), os adultos de Hedylidae possuem corpo extremamente delgado, antenas filiformes ou bipectinadas, asas anteriores com ápice levemente emarginado e de coloração castanha ou cinza, mas algumas espécies são predominantemente ou inteiramente brancas; possuem estruturas de acoplamento, frênulo e retináculo, sendo este último ausente nas fêmeas. Outra característica marcante nessa família é a presença de órgãos timpânicos no primeiro par de asas (Scoble, 1986).

Os adultos são capturados, em geral, com armadilhas luminosas. Podem ser coletados desde o nível do mar até altitudes de 3.000 metros (Scoble, 1990b; Scoble e Aiello, 1990; Scoble, 1995).

Ocorrem principalmente em áreas de floresta primária (Scoble, 1990b). Assim, estão vulneráveis às mudanças ambientais, especialmente aquelas de caráter antrópico.

Com objetivo de contribuir para um melhor conhecimento da diversidade de Hedylidae da Amazônia brasileira, foi efetuado o registro das espécies que voam no dossel de floresta de terra firme a partir de coletas noturnas na Torre do $\mathrm{km} 14$, do núcleo ZF-2.

\section{MATERIAL E MÉTODOS}

O material estudado foi coletado em uma torre de $50 \mathrm{~m}$ de altura, localizada no $\mathrm{km} 14$ da vicinal ZF-2 $\left(2^{\circ} 35^{\prime} 21^{\prime \prime} \mathrm{S} / 60^{\circ} 06^{\prime} 55^{\prime \prime} \mathrm{W}\right)$, Estação Experimental de Silvicultura Tropical do INPA, que tem uma área de 21.000 ha e fica dentro do Distrito Agropecuário da SUFRAMA (Superintendência da Zona Franca de Manaus), tendo como limites a Rodovia BR-174 (Manaus - Boa Vista) e a bacia do rio Cuieiras.
Segundo o RADAM/BRASIL (1978), trata-se de uma área de terra firme coberta por uma Floresta Densa Tropical; o clima é do tipo "Am", segundo a classificação de Köppen, corresponde a um clima tropical quente e úmido, com chuvas abundantes e bem distribuídas ao longo do ano; temperatura média anual de $26^{\circ} \mathrm{C}$, com a mínima de $18^{\circ} \mathrm{C}$ e a máxima de $39^{\circ} \mathrm{C}$; a precipitação anual varia de 1.800 a $2.800 \mathrm{~mm}$.

Foram realizadas coletas mensais, de janeiro a dezembro de 2004, por três noites consecutivas, durante as Luas Minguante e/ou Nova, das 18:00 às 06:00 h, registrando o intervalo de hora da chegada de cada espécime à luz. Alguns exemplares foram capturados em coletas preliminares que ocorreram em janeiro de 1997, outubro e novembro de 2003.

Foi utilizado um lençol branco, medindo 1,40 x 2,20 m, iluminado por uma lâmpada de luz mista de mercúrio de 250W e uma lâmpada de luz negra UV-BLB no sentido lesteoeste, a $40 \mathrm{~m}$ de altura na torre. Os insetos foram coletados manualmente, mortos e acondicionados individualmente em envelopes entomológicos.

Os espécimes foram identificados com auxílio das descrições originais e por comparações com diagnoses e figuras, tomando como base os trabalhos de Prout (1932) e Scoble (1990a, b).

\section{RESULTADOS}

Foram obtidos 55 exemplares pertencentes a nove espécies, sendo uma espécie não identificada, provavelmente uma espécie nova (Tabela 1).

Considerando todos os hedilídeos coletados em 2004, $M$. hedylaria foi a mais abundante com $36,4 \%$, seguida de $M$. bahiata e $M$. nigrimacula com $16,4 \%$ e $10,9 \%$, respectivamente (Tabela 1). A maioria dos exemplares obtidos,

Tabela 1 - Abundância das espécies de Hedylidae, por sexo, coletadas a $40 \mathrm{~m}$ de altura, na torre do km 14, núcleo ZF-2, ano de 2004 na Estação Experimental de Silvicultura Tropical, Manaus, Amazonas, Brasil.

\begin{tabular}{lllll}
\hline Espécies & $\begin{array}{l}\mathbf{N}^{0} \text { de } \\
\text { Machos }\end{array}$ & $\begin{array}{l}\mathbf{N}^{\mathbf{0}} \text { de } \\
\text { Fêmeas }\end{array}$ & $\begin{array}{l}\mathbf{N}^{\mathbf{0}} \text { de } \\
\text { exemplares }\end{array}$ & $\begin{array}{l}\text { Abundância } \\
(\%)\end{array}$ \\
\hline M. bahiata & 9 & & 9 & 16,4 \\
\hline M. conifera & 3 & & 3 & 5,5 \\
\hline M. hedylaria & 19 & 1 & 20 & 36,4 \\
\hline M. heliconiaria & 1 & 1 & 2 & 3,6 \\
\hline M. hyacinthina & 4 & & 4 & 7,3 \\
\hline M. klagesi & 5 & & 5 & 9,1 \\
\hline M. lucivittata & 2 & 2 & 3,6 \\
\hline M. nigrimacula & 6 & & 6 & 10,9 \\
\hline Macrosoma sp. & 4 & & 4 & 7,3 \\
\hline TOTAL & & & 55 & 100,0 \\
\hline
\end{tabular}


$95 \%$, foi de machos. As fêmeas correspondem às espécies $M$. hedylaria e M. heliconiaria.

Ocorreu maior número tanto de indivíduos quanto de espécies na estação mais chuvosa, notadamente nos meses de fevereiro, abril e maio (Figura 1). A distribuição anual, por espécie, dos exemplares coletados em 2004 é apresentada na Tabela 2.

Os hedilídeos apresentaram atividade de vôo desde a primeira hora da noite até a última hora da madrugada, com maior abundância no intervalo de 19:00 às 22:00h, intervalo que coletou $80 \%$ dos espécimes. A partir de 22:00h o rendimento da coleta decresce significativamente (Figura 2). $M$. hedylaria foi a única espécie para qual se observou uma relativa preferência pelo intervalo hora de 19:00 às 20:00h, pois 12 exemplares foram coletados nesse intervalo hora (Tabela 3), esse total corresponde à $60 \%$ dos indivíduos dessa espécie.

Como resultado das coletas preliminares em janeiro de 1997 foram capturados um exemplar de M. nigrimacula

Tabela 2 - Distribuição anual das espécies de Hedylidae, coletadas a $40 \mathrm{~m}$ de altura, na torre do km 14, núcleo ZF-2, ano de 2004 na Estação Experimental de Silvicultura Tropical, Manaus, Amazonas, Brasil.

\begin{tabular}{|c|c|c|c|c|c|c|c|c|c|c|c|c|}
\hline \multirow[b]{2}{*}{ Espécie } & \multicolumn{12}{|c|}{ Meses } \\
\hline & Jan & Fev & Mar & Abr & Mai & Jun & Jul & Ago & Set & Out & Nov & Dez \\
\hline M. bahiata & & & & 1 & 1 & 2 & 1 & & 2 & & & 2 \\
\hline M. conífera & & & & 1 & 1 & & 1 & & & & & \\
\hline M. hedylaria & 2 & 3 & & 5 & 3 & & 1 & 1 & & 1 & 1 & 3 \\
\hline M. heliconiaria & & 1 & & & 1 & & & & & & & \\
\hline M. hyacinthina & & 1 & & 1 & & 1 & & & 1 & & & \\
\hline M. klagesi & & & 2 & & 1 & & 1 & & & 1 & & \\
\hline M. Iucivittata & & & & & 1 & & & 1 & & & & \\
\hline M. nigrimacula & & 2 & & & 1 & & & & & 1 & 1 & 1 \\
\hline Macrosoma sp. & & 2 & & & & & & & 1 & 1 & & \\
\hline
\end{tabular}

e dois de $M$. hedylaria, todos machos. Em 2003 foram coletados exemplares únicos de $M$. klagesi, $M$. conifera, $M$. hedylaria e M. nigrimacula, machos. Em novembro de 2003 foram capturados um macho de $M$. conifera, um de $M$. hedylaria e outro de Macrosoma sp., além de uma fêmea de M. lucivittata.

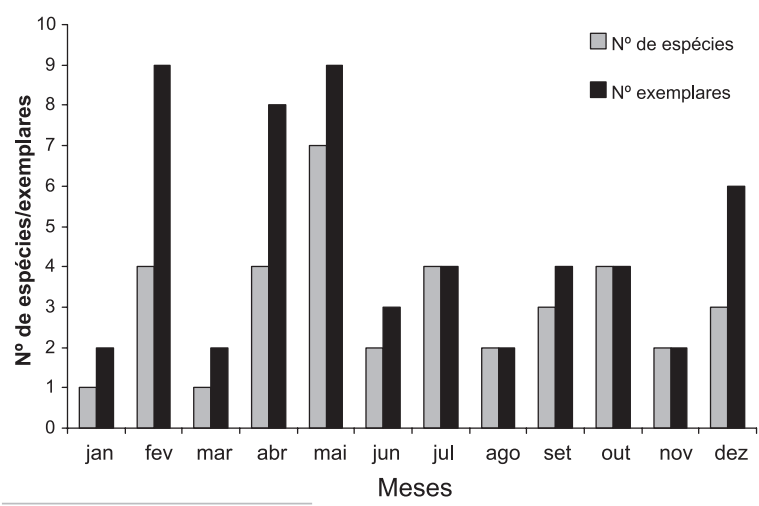

Figura 1- Número de espécies e exemplares de Hedylidae coletados em 2004, a $40 \mathrm{~m}$ de altura, na torre do km 14, núcleo ZF-2, estação Experimental de Silvicultura Tropical, Manaus, Amazonas, Brasil.

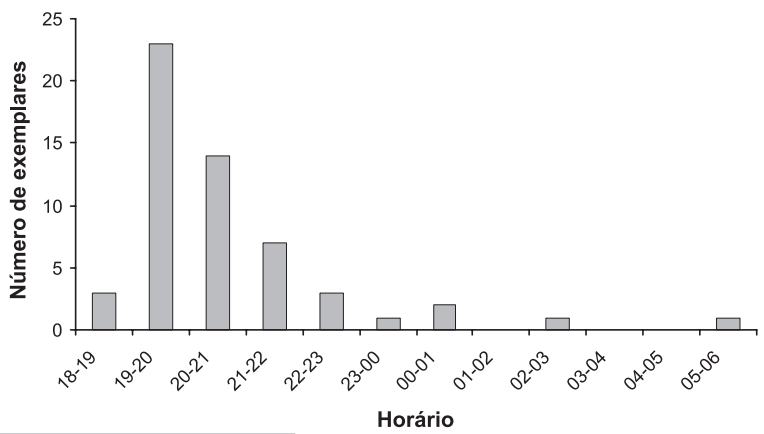

Figura 2- Número de exemplres de Hedylidae capturados por intervalo hora em 2004, a 40 m de altura, na torre do km 14, núcleo ZF-2, estação Experimental de Silvicultura Tropical, Manaus, Amazonas, Brasil.

Tabela 3 - Distribuição por intervalo de hora das espécies de Hedylidae, coletadas a 40 m de altura, na torre do km 14, núcleo ZF-2, ano de 2004 na Estação Experimental de Silvicultura Tropical, Manaus, Amazonas, Brasil.

\begin{tabular}{|c|c|c|c|c|c|c|c|c|c|c|c|}
\hline \multirow{2}{*}{ Espécie } & \multicolumn{11}{|c|}{ Intervalos de hora } \\
\hline & $18-19$ & $19-20$ & $20-21$ & 21-22 & $22-23$ & 23-00 & $00-01$ & 01-02 & $02-03$ & 04-05 & $05-06$ \\
\hline M. bahiata & & 2 & 4 & & 1 & & 1 & & 1 & & \\
\hline M. conífera & & 2 & & & & 1 & & & & & \\
\hline M. hedylaria & 3 & 12 & 3 & 2 & & & & & & & \\
\hline M. heliconiaria & & & & 1 & & & 1 & & & & \\
\hline M. hyacinthina & & 2 & 1 & 1 & & & & & & & \\
\hline M. klagesi & & & 4 & 1 & & & & & & & \\
\hline M. lucivittata & & & 1 & 1 & & & & & & & \\
\hline M. nigrimacula & & 5 & & & 1 & & & & & & \\
\hline Macrosoma sp.* & & & 1 & 1 & 1 & & & & & & 1 \\
\hline
\end{tabular}




\section{DISCUSSÃO}

As espécies identificadas representam $23 \%$ das espécies descritas, $38 \%$ das espécies registradas para o Brasil e 50\% daquelas com registro para Amazônia brasileira. A coleta de nove espécies de Hedylidae, em uma área restrita como a torre da ZF-2 é relativamente alta se comparada aos levantamentos realizados em grandes áreas como a Costa Rica com 14 espécies (Scoble, 1992), Panamá com nove (Aiello, 1992), Peru com 26 (Lamas e Grados, 1997) e Equador com 15 espécies (Piñas e Manzano, 2001).

Os dados apresentados são inéditos para esse grupo, especialmente no dossel de floresta de terra firme, e representam um indício de que as espécies voam sobre as copas das árvores seja para dispersão, oviposição ou procura de alimento. A influência atrativa sobre eventuais espécimes voando nos estratos inferiores foi mínima, pois a própria copa das árvores serve de anteparo para os feixes de luz e, mesmo a parte oeste da torre estando relativamente exposta, a dispersão dos raios luminosos da armadilha, a $40 \mathrm{~m}$ de altura, não se propaga a mais de $50 \mathrm{~m}$ de diâmetro no solo. De acordo com Janzen (1983), as mariposas são "atraídas" à luz só de distâncias relativamente curtas.

O maior número de espécies e exemplares coletados, coincide com os meses do período mais chuvoso na região. Não foi possível avaliar regularidade sazonal para as espécies capturadas, porém para $M$. hedylaria, a mais abundante, foi observada ausência apenas nos meses de março, junho e setembro.

Com base em trabalhos sobre coletas de insetos com armadilha luminosa, Southwood (1975) relata que o maior número de machos em relação às fêmeas pode ser devido a um efeito seletivo da qualidade da luz sobre os sexos.

Silveira Neto et al. (1976), por outro lado, sugerem que uma das possíveis razōes para o maior número de machos é que as fêmeas, por serem mais pesadas, voem menos que estes, especialmente se estiverem fecundadas. Estes autores sugerem ainda que em alguns grupos de lepidópteros, as fêmeas, quando fecundadas, saem em busca da planta alimento para as larvas, por isso tendem a ser menos sensíveis à luz.

A atividade de vôo dos hedilídeos foi mais intensa no período de 19:00 às 22:00h, considerando o número total de exemplares coletados em 2004. O decréscimo na atividade de vôo ao longo da noite pode estar relacionado à redução da temperatura, fator observado por Laroca e Mielke (1975) ao estudarem a ecologia de comunidade de Sphingidae no Paraná.

\section{AGRADECIMENTOS}

Aos Drs. Niro Higuchi e Joaquim dos Santos pelos valiosos auxílios durante a realização deste trabalho. À Bióloga Simone
Ferreira Trovisco e ao Alexandre da Silva Filho pelo auxílio durante as coletas dos Hedylidae, ao funcionário José Maria Bezerra da Paz e aos vigilantes da Reserva pelo apoio prestado à equipe.

\section{BIBLIOGRAFIA CITADA}

Aiello, A. 1992. Nocturnal butterflies in Panama, Hedylidae (Lepidoptera: Rhopalocera) In: Quintero, D.; Aiello, A. (Eds.). Insects of Panama and Mesoamerica: Selected Studies. Oxford University Press, London. p. 549-553.

Druce, H. 1893. Geometridae. Hedylinae. In: Porter, R.H. (Ed.). Biologia Centrali-Americana. Insecta. Lepidoptera-Heterocera. Bernard Quaritch, London. p. 178-179.

Janzen, D.H. 1983. Insects. In: Janzen, D.H. (Ed.). Costa Rican Natural History. The University of Chicago Press, Chicago and London. p. 619-779. Lamas, G.; Grados, J. 1997. Sinopsis de los Hedylidae (Lepidoptera) del Perú. Revista peruana de Entomologia, 40: 107-109.

Lamas, G. \& J. GRADOS. 1997. Sinopsis de los Hedylidae (Lepidoptera) del Perú. Revista Peruana de Entomologia, 40: 107-109

Laroca, S.; Mielke, O.H.H. 1975. Ensaios sobre ecologia de comunidade em Sphingidae na Serra do Mar, Paraná, Brasil (Lepidoptera). Revista Brasileira de Biologia, 35 (1): 1-19.

Piñas, F.; Manzano, I. 2001. Hedylidae Macrosoma. Revista Ecuatoriana de Mariposas, 9: 1-7.

Prout, L.B. 1916. New Neotropical Geometridae. Novitates Zoologicae, 23 (2): 151-190.

Prout, L.B. 1932. The American Geometridae In: Seitz, A. (Ed.). The Macrolepidoptera of the World, Vol. 8. p. 16-20.

RADAM/BRASIL 1978. Programa de Integração Nacional. Levantamento de recursos Naturais. V.18 (Manaus) - RADAM (Projeto) DNP, Ministério de Minas e Energia. Brasil. 626pp.

Scoble, M.J. 1986. The structure and affinities of the Hedyloidea: a new concept of the butterflies. Bulletin of the British Museum Natural History (Entomology), 53(5): 251-286.

Scoble, M.J. 1990a. A catalogue of the Hedylidae (Lepidoptera: Hedyloidea), with descriptions of two new species. Entomologica Scandinavica, 21: 113-119.

Scoble, M.J. 1990b. An identification guide to the Hedylidae (Lepidoptera: Hedyloidea). Entomologica Scandinavica, 21: 121-158.

Scoble, M.J. 1992. Guia de las mariposas hedylidas de Costa Rica (Lepidoptera: Hedylidae). INBIO, Heredia-Costa Rica. 30pp.

Scoble, M.J. 1995. The Lepidoptera: Form, Function and Diversity. The Natural History Museum, London \& Oxford University Press. 440pp.

Scoble, M. 1998. Hedylidae. In Heppner, J.B. (Ed.). Lepidopterorum Catalogus (New Series), 93. viii+8pp.

Scoble, M.J.; Aiello, A. 1990. Moth-like butterflies (Hedylidae: Lepidoptera): a summary, with comments on the egg. Journal of Natural History, 24: 159-164.

Silveira Neto, S.; Nakano, O.; Barbin, D.; Villa Nova, N.A. 1976. Manual de Ecologia dos Insetos. S. Paulo, Agronômica Ceres, 419pp.

Southwood, T.R.E. 1975. Ecological Methods. Chapman \& Hall, London. 391pp.

Recebido em 13/12/2005

Aceito em 10/10/2007 